\title{
Relationship of general trust with individual health and life related factors among frail elderly residents at home in Hokkaido rural areas in Japan
}

\author{
Motoyuki Yuasa $^{1^{*}}$, Tamiko Ikeno ${ }^{2}$, Shigekazu Ukawa ${ }^{3}$ \\ ${ }^{1}$ Department of Public Health, Graduate School of Medicine, Juntendo University, Tokyo, Japan; \\ *Corresponding Author: moyuasa@juntendo.ac.jp \\ ${ }^{2}$ Center of Environmental and Health Sciences, Hokkaido University, Sapporo, Japan \\ ${ }^{3}$ Department of Public Health Sciences, Graduate School of Medicine, Hokkaido University, Sapporo, Japan
}

Received 5 March 2012; revised 3 April 2012; accepted 10 April 2012

\section{ABSTRACT}

General Trust (GT), defined as a default expectation of other people's trustworthiness, is assumed to be a predictor for promotion of health and welfare in individuals as well as for strengthening of social capital in the community. An improvement of health and quality of life of the elderly is recently a crucial agenda. Thus, the purpose of the study was to explore which factors regarding health and life associated with GT among frail elderly people living at homes in Japanese rural area. The study selected the subjects who were designated within three mildest degrees in seven stages of long-term care levels and who met at an item and more in the national basic check-list. 209 participants were interviewed by trained health personnel using a structured questionnaire. GT was measured by a dichotomous outcome of inquiring "do you trust people in general?". In the univariable analysis, educational status $(p=0.004)$, activity competence index including instrumental activity of daily living (IADL) $(p=0.020)$, Mini-Mental State Examination (MMSE) $(p=0.029)$ and Self-rating Depression Scale (SDS) $(p=0.010)$ were significantly related to GT. By logistic regression analysis using a stepwise method with a likelihood ratio, educational status alone was significantly associate with GT $(p=0.010$, odds ratio $=1.195[95 \%$ confidence interval; 1.043 1.371]). Health related factors had nothing to do with GT. Our finding suggested that the higher educated elderly might have had more opportunities to encounter the others and more indispensability to deliberately discern their trustworthiness than the lower, and consequently had higher GT through social intelligence being strengthened.

Keywords: General Trust; Social Capital; Aged; Long-Term Care; Japan

\section{INTRODUCTION}

Recently, researchers across a wide range of academic fields have devoted attention to general trust (GT). Sociologists and economists regard GT as an interpersonal phenomenon or a game-theoretical behavior $[1,2]$, and psychologists view GT as a personality trait [3]. GT has been also investigated as a natural scientific concern in psychosocial and physiological areas [4,5]. As GT is an inter-disciplinary concept, with cross-cultural and crosslevel dimensions, many definitions exist. Yamagishi \& Yamagishi [6] defined GT as an expectation of benign or cooperative behavior based on the goodwill of the partner, or more simply, as the default expectation of other people's trustworthiness [7]. Other researchers state that GT focuses on potential risk-taking behavior and define GT as the extent to which one believes that others will not act to exploit one's vulnerabilities [8-10]. Although there are many different ways of conceptualizing trust, GT measured by inquiring "do you trust others in general?" is distinguished from information-based trust (IBT) in which one discerns the trustworthiness of a single person based on specific information provided.

While it has been crucial to enhance human capability as human capital, the strengthening of social capital has also been well understood to be an indispensable factor in any development fields. Social capital refers to the relationship between people such as in social networks, social support, norms, and mutual aid. GT is a key component of cognitive social capital. As social capital is thought to relate to health and quality of life [11-13], GT may predict not only the health and welfare of individu- 
als but also of the development of a community. Recently industrialized countries, and even some developing nations, have begun to focus on improving the health and welfare of elderly individuals as well as on strengthening social capital by providing a supportive community for the elderly [14]. However, little research has demonstrated the relationship between GT and individual characteristics, health, and life-related factors in the elderly. Therefore, we conducted the study by logistic regression analysis using cross-sectional data, aiming to explore which factors were associated with GT among frail elderly people living in their homes in a rural area in Hokkaido, in the northern region of Japan.

\section{METHODS}

\subsection{Participants}

Study participants were recruited from two rural towns, Hidaka and Shin-Hidaka, in Hokkaido Prefecture, with a total population of 12,600 and 27,000 in 2008, respectively. Approximately $24 \%$ of residents of both towns were older than 65 years. The main industries in the towns were breeding of thoroughbred horses and a fishery for Hidaka kelp. Subjects aged 65 years and older who were living at home and met the following inclusion criteria were included in the study: 1) within the three mildest of seven stages of long-term care levels, assigned through a public assessment tool used by the national long-term care insurance system to provide public services; and 2) met one or more items in the basic checklist devised by the Japanese Ministry of Health, Labor and Welfare to assess the level of care needed for the elderly. Subjects who scored less than 17 on the minimental state examination (MMSE) $[15,16]$ or had medical diagnosis due to dementia or depression were excluded from the analysis because their intelligence level could make them difficult to interview. This study was performed as a sub-research of a survey to assess physical and cognitive function among frail elderly living at home.

\subsection{Procedure}

We trained health and long-term care personnel as the interviewers during a half-day session on standard procedures and interviewing skills. Participants were interviewed during a home visit by the trained interviewer using a structured questionnaire. GT was measured by a dichotomous outcome with the simple question, "do you trust people in general?" We measured individual sociodemographics, health, and socioeconomic factors such as gender; age; educational status by year; degree of longterm care; self-rated health [17]; activity competence index including instrumental activities of daily living
(IADL), developed by the Tokyo Metropolitan Institute of Gerontology (TMIG); MMSE [18]; self-rating depression scale (SDS) [19]; general self efficacy score (GSES) [20]; use of private and/or public care services; medical history; visual and hearing disorders; family structure; communication with family and neighbors; satisfaction with neighbors; hobbies; current job; annual income; and satisfaction with income.

Degree of care was assessed by a public assessment tool devised by the Japanese Ministry of Health, Labor and Welfare. Public or private services were measured using a response of yes and no to use. Self-rated health was a perceived general health condition that was measured by asking "would you say that in general your health is excellent, very good, good, fair, or poor?" The activity competence index, including IADL, was measured through 13 questions on IADL, intellectual activity, and social participation with responses ranging from 0 to 13; higher scores mean a better activity. MMSE included 11 questions with total scores ranging from 0 to 30 ; higher scores indicate a better mental condition. SDS was assessed using 20 items; total scores ranged from 20 to 80 with higher scores indicating a greater level of depression. GSES is a validated generalized self-efficacy scale that includes 10 questions scored using four Likert scores and has the possible total range of scores from 0 to 40 ; lower scores mean a stronger self-efficacy. Visual and hearing disorder was assessed by a response indicating "not at all", "no disorder with glasses or a hearing aid" and "still disorder even with glasses or a hearing aid". Family structure was determined by living with alone, partner, children or both of partner and children. Satisfaction with neighborhood and income was measured by a response indicating "satisfied", "not satisfied" and "difficult to answer". Hobby and current job were determined using yes and no responses. The interview spent less than 45 minutes with each subject.

All subjects were informed of the study objective and procedures before the interview, and provided written informed consent. The study protocol was approved by the ethics board for epidemiological studies at Hokkaido University Graduate School of Medicine. The study protocol conformed to the Helsinki Declaration and the ethical rules of the Japanese Ministry of Health, Labor and Welfare.

\subsection{Statistical Analysis}

The association between GT and different variables was determined using univariate analysis that included chi-square analysis with Fisher's adjustment or MannWhitney tests. Variables with a $p$ value $\leq 0.2$ at univariate analysis were included in the logistic regression analysis with a binominal variable: can trust or can NOT 
trust. To explore variables that were significantly associated with GT and determine odds rations and its 95\% confidence intervals, we conducted a compulsive injection method as well as a stepwise method with a likelihood ratio was used. Subsequently, Spearman's correlation coefficient was calculated between the significant variables obtained at the above analysis. Results were considered statistically significant if the $p$ value was $<0.05$. Statistical analyses were performed using SPSS ver. 17.0 for Windows (SPSS Inc., Chicago, IL, USA).

\section{RESULTS}

Of 252 participants who agreed to participate in the study, 209 subjects were included in the final analysis because 43 subjects excluded was based on MMSE score lower than 17. Table 1 shows characteristics of subjects and results of univariate analysis between GT and different variables. Subjects had a mean age of $77.7 \pm 7.5$ years (range, 65 to 95 years). Two thirds of subjects attained 9 years or less of compulsory education in Japan. A quarter of the subjects had a primary school education level; $8.2 \%$ had reached the level of college or higher. Half of subjects had used public care services provided by the national long-term care insurance system because they were physically frail. Thirty percent of the elderly rated their health as poor, and almost $90 \%$ suffered from some disease. More than a third lived alone $(36.9 \%)$ or with a partner $(34.6 \%)$; seven subjects $(4.3 \%)$ indicated they had no relationship with family who lived separately. Approximately $40 \%$ still worked. More than half (60.2\%) had annual income of less than 1.5 million Japanese yen, nearly equivalent to US $\$ 18,750$ as of the end of 2010 ; $39.6 \%$ indicated that they were satisfied with their income. Sex, educational status, degree of care, use of public care services, competence index on IADL of TMIG, MMSE, SDS, and relationship with family living separately were significantly associated with GT ( $p \leq$ 0.2 ). These variables were included in the multivariate analysis.

Table 2 shows the results of two logistic regression analyses. Although there was no significant factor associated with GT in the compulsive injection method, the stepwise method with likelihood ratios indicated that educational status was significantly related to GT ( $p=$ $0.010 ; \mathrm{OR}=1.195[95 \% \mathrm{CI}, 1.043-1.371]$ ). The degree of care registered in the long-term care insurance system and the use of public services were excluded from the analysis due to the probability of multicollinearity with the activity competence index, including IADL of TMIG, because both valuables were officially determined to depend partly on the level of the activity competence index (data not shown). The average years of educational duration of those who answered yes or no regarding GT were $9.3 \pm 2.9$ and $7.7 \pm 2.7$, respectively (Table $\mathbf{1}$ ), which was significantly different $(p=0.002)$ by MannWhitney tests.

Table 1. Characteristics of the subjects and the results of univariate analysis between GT and different covariates.

\begin{tabular}{|c|c|c|c|c|}
\hline Covariates & $\mathrm{N}(\%)$ & Can trust & Can NOT trust & $p^{\mathrm{a}}$ \\
\hline \multicolumn{5}{|l|}{ Basic characteristics } \\
\hline Sex & & & & 0.074 \\
\hline Male & $69(33.0)$ & 55 & 14 & \\
\hline Female & $140(67.0)$ & 94 & 46 & \\
\hline Age (years) ${ }^{b}$ & $77.7 \pm 7.5$ & $77.7 \pm 7.5$ & $77.7 \pm 7.5$ & 0.935 \\
\hline Educational status (years) ${ }^{\mathrm{b}}$ & $8.9 \pm 2.9$ & $9.3 \pm 2.9$ & $7.7 \pm 2.7$ & 0.004 \\
\hline$\leq 6$ & $44(25.7)$ & 28 & 16 & \\
\hline $7-9$ & $72(42.1)$ & 51 & 21 & \\
\hline $10-12$ & $41(24.0)$ & 35 & 6 & \\
\hline$\geq 13$ & $14(8.2)$ & 13 & 1 & \\
\hline \multicolumn{5}{|l|}{ Long-term care } \\
\hline Degree of care & & & & 0.189 \\
\hline Slightly frail elderly & $74(35.4)$ & 58 & 16 & \\
\hline Frail elderly & $9(4.3)$ & 8 & 1 & \\
\hline Needs assistance-level 1 & $38(18.2)$ & 24 & 14 & \\
\hline Needs assistance-level 2 & $40(19.1)$ & 30 & 10 & \\
\hline Needs long-term care-level 1 & $48(23.0)$ & 29 & 19 & \\
\hline Public services on long-term care insurance & & & & 0.061 \\
\hline used & $90(50.8)$ & 61 & 29 & \\
\hline NOT used & $87(49.2)$ & 70 & 17 & \\
\hline
\end{tabular}




\section{Continued}

\begin{tabular}{|c|c|c|c|c|}
\hline Private services on long-term care & & & & 0.668 \\
\hline used & $34(19.0)$ & 24 & 10 & \\
\hline NOT used & $145(81.0)$ & 108 & 37 & \\
\hline \multicolumn{5}{|l|}{ Health } \\
\hline Self-rated health & & & & 0.654 \\
\hline very good & $13(6.2)$ & 9 & 4 & \\
\hline good & $35(16.7)$ & 24 & 11 & \\
\hline fair & $93(44.5)$ & 68 & 25 & \\
\hline poor & $57(27.3)$ & 41 & 16 & \\
\hline very poor & $8(3.8)$ & 4 & 4 & \\
\hline unknown & $3(1.4)$ & 3 & 0 & \\
\hline Activity competence index including IADL of TMIG ${ }^{\mathrm{b}}$ & $9.4 \pm 3.3$ & $9.7 \pm 3.3$ & $8.7 \pm 3.2$ & 0.020 \\
\hline $\mathrm{MMSE}^{\mathrm{b}}$ & $25.2 \pm 3.2$ & $25.5 \pm 3.2$ & $24.5 \pm 3.3$ & 0.029 \\
\hline $\mathrm{SDS}^{\mathrm{b}}$ & $36.7 \pm 9.1$ & $35.6 \pm 8.9$ & $39.1 \pm 9.2$ & 0.010 \\
\hline General self efficacy score ${ }^{b}$ & $18.9 \pm 7.5$ & $18.5 \pm 7.1$ & $19.7 \pm 8.4$ & 0.611 \\
\hline Comorbidities & & & & 0.582 \\
\hline have & $159(89.3)$ & 119 & 40 & \\
\hline NOT have & $19(10.7)$ & 13 & 6 & \\
\hline Visual disorder & & & & 0.516 \\
\hline have NO disorder & $64(36.4)$ & 50 & 14 & \\
\hline have NO disorder with glasses & $79(44.9)$ & 58 & 21 & \\
\hline have disorder even if with glasses & $33(18.8)$ & 22 & 11 & \\
\hline Hearing disorder & & & & 0.418 \\
\hline have NO disorder & $141(80.1)$ & 107 & 34 & \\
\hline have NO disorder with a hearing aid & $4(2.3)$ & 2 & 2 & \\
\hline have disorder even if with a hearing aid & $31(17.6)$ & 20 & 11 & \\
\hline \multicolumn{5}{|l|}{ Current life } \\
\hline Whom living with & & & & 0.648 \\
\hline living alone & $66(36.9)$ & 49 & 17 & \\
\hline living with partner & $62(34.6)$ & 45 & 17 & \\
\hline living with children & $22(12.3)$ & 15 & 7 & \\
\hline living with partner and children & $17(9.5)$ & 12 & 5 & \\
\hline other & $12(6.7)$ & 11 & 1 & \\
\hline Relationship with family living separately & & & & 0.083 \\
\hline have relationship & $154(95.7)$ & 115 & 39 & \\
\hline have NO relationship & $7(4.3)$ & 3 & 4 & \\
\hline Satisfaction with the neighborhood & & & & 0.424 \\
\hline satisfied & $141(78.8)$ & 107 & 34 & \\
\hline NOT satisfied & $7(3.9)$ & 5 & 2 & \\
\hline difficult to answer & $31(17.3)$ & 20 & 11 & \\
\hline Hobby & & & & 0.302 \\
\hline have & $100(56.8)$ & 77 & 23 & \\
\hline NOT have & $76(43.2)$ & 53 & 23 & \\
\hline \multicolumn{5}{|l|}{ Economics } \\
\hline Current job & & & & 0.864 \\
\hline have & $73(41.2)$ & 53 & 20 & \\
\hline NOT have & $104(58.8)$ & 77 & 27 & \\
\hline Annual income in Japanese yen & & & & 0.505 \\
\hline$<1,000,000$ & $69(43.7)$ & 45 & 24 & \\
\hline $1,000,000-1,500,000$ & $26(16.5)$ & 22 & 4 & \\
\hline $1,500,000-2,000,000$ & $21(13.3)$ & 17 & 4 & \\
\hline $2,000,000-2,500,000$ & $20(12.7)$ & 16 & 4 & \\
\hline$\geq 2,500,000$ & $22(13.9)$ & 17 & 5 & \\
\hline Satisfaction with the income & & & & 0.607 \\
\hline satisfied & $67(39.6)$ & 50 & 17 & \\
\hline NOT satisfied & $56(33.1)$ & 39 & 17 & \\
\hline difficult to answer & $46(27.2)$ & 36 & 10 & \\
\hline
\end{tabular}

${ }^{\mathrm{a}} \chi^{2}$ test, applied Fisher's adjustment, and Mann-Whitney; ${ }^{\mathrm{b}}$ mean \pm standard deviation. 
Table 2. Odds ratio between GT and covariates in logistic regression analysis.

\begin{tabular}{lcccc}
\hline Covariates & $\beta$ & SD & $p$ & $\begin{array}{c}\text { OR (95\% Interval Confi- } \\
\text { dence) }\end{array}$ \\
\hline $\begin{array}{l}\text { By compulsive injection method } \\
\quad \text { Sex }^{\mathrm{a}}\end{array}$ & -0.674 & 0.553 & 0.223 & $1.962(0.663-5.804)$ \\
$\quad$ Activity competence index including IADL of TMIG & 0.099 & 0.074 & 0.178 & $1.104(0.956-1.276)$ \\
MMSE & -0.045 & 0.078 & 0.565 & $0.956(0.820-1.114)$ \\
SDS & -0.040 & 0.025 & 0.112 & $0.961(0.915-1.009)$ \\
$\quad$ Relationship with family living separately & -0.009 & 0.980 & 0.992 & $0.991(0.145-6.758)$ \\
$\quad$ Educational status (years) & 0.099 & 0.091 & 0.275 & $1.105(0.924-1.321)$ \\
By stepwise method with a likelihood ratio & & & & \\
$\quad$ Educational status (years) & 0.179 & 0.070 & 0.010 & $1.195(1.043-1.371)$ \\
\hline
\end{tabular}

${ }^{\mathrm{a}}$ reference $=$ male; ${ }^{\mathrm{b}}$ reference $=$ those who had no relationship with family living separately.

Table 3 shows the significant items in the Spearman's correlation analysis that examined associations between educational status and variables. Age was negatively associated with educational status, and activity competence index including IADL of TMIG, MMSE, and annual income were positively associated with educational status.

\section{DISCUSSION}

Results of logistic regression analysis suggested that GT was significantly associated with educational status among frail elderly who lived at home in a rural Japanese area. Elderly subjects who answered "yes" to the question "do you trust people in general?" had a $20 \%$ higher educational status than those responding "no." Ichida et al. conducted a study in Japan and reported the same link between education and GT; however, they did not discuss this finding in detail [21]. The following review of previous studies relevant to the psychological and empirical theory of GT developed by Yamagishi et al. [22] helps interpret the meaning of the association between GT and educational status in our study.

In a cross-societal questionnaire study between the United States and Japan, Yamagishi and Yamagishi [6] found that American subjects had a higher level of GT than Japanese subjects. Using the cultural configuration of a Japanese society that is often characterized as a collectivism [23] and the concept of "social uncertainty" defined as people's inability to detecting partners' intentions correctly [24], Yamagishi et al. [22] predicted that GT may be necessary for people to interact with others in a society like the United States, where there is much more social uncertainty. On the other hand, in a society with little social uncertainty and a collective like Japan, less GT may be required. Fukuyama [25] also argued that a society based on the collective with robust ties of family and neighbors is unlikely to promote GT beyond family and acquaintances. Yamagishi [6] recognized that in societies with higher social uncertainty, people may require a "commitment formation" with others to reduce
Table 3. Significant Spearman's correlation with educational status.

\begin{tabular}{lc}
\hline & Spearman's coefficient \\
\hline Age (years) & -0.601 \\
Activity competence index including & 0.430 \\
IADL of TMIG & 0.473 \\
MMSE & -0.227 \\
SDS & -0.309 \\
General self efficacy score & 0.444 \\
Annual income (Japanese yen)
\end{tabular}

uncertainty in relationships; this commitment formation mitigates uncertainty in a relationship. However, this commitment formation depends on individuals staying in the committed relationship once it has been established. If they want to leave the current relationship and explore other opportunities, they need to pay more attention and more carefully process information concerning signals of trustworthiness of others. They must work harder to improve "social intelligence," which is defined as the ability to understand one's own and other people's internal states and use such understanding in social relationships. Thus, people need to be able to use social intelligence to discern the trustworthiness of a potential partner; GT is a by-product of this process [7]. Yamagishi et al. [6] hypothesized that a high level of GT makes people more willing to venture into a socially uncertain world in pursuit of better opportunities and that such actions occur more frequently in the United States than in Japan. He referred to this as the emancipation theory, indicating that GT emancipates people from the confines of the security of stable relationships. He also indicated that GT is generated by social intelligence and can be strengthened by increased experiences of human relationships with many strangers.

Our study participants graduated from schools around the Second World War. In rural areas of Japan during this time, it is likely that those with less education had limited employment opportunities and could not seek jobs 
outside their living area. As a result, a lower educational status kept people from going outside of their limited relationships and having the opportunity to increase their acquaintanceships in the outside world. According to the leading theory of Yamagishi et al. [22], those with lower educational status might be inclined to regard all strangers as untrustworthy and avoid interacting with outsiders if possible. Therefore, they had no need to develop GT. In contrast, a higher educational level might encourage people to go out and look for modern, skilled work. This opportunity would lead to additional experiences in human relationships with diverse outsiders compared with people with a lower education. This opportunity strengthens their social intelligence and GT.

Our findings demonstrated a significant association between GT and educational status. According to Table 3, the high correlation with educational status was supposed to account for the association with competence index on IADL of TMIG and MMSE as well as a higher annual income. In general, higher educated people tend to obtain an occupation providing a higher income. Yamagishi et al. [22] indicated that a higher education disposes people to have a social intelligence that stimulates physical competence and cognitive function. Table 3 shows a negative correlation between educational status and age because the study subjects were so old, and these older participants might have had less opportunity to complete their school education during the era of the Second World War.

Information bias is often involved in GT assessment studies unless a distinction between GT and IBT is considered. IBT is a trust toward a specified person based on particular information relevant to that person. Our study had no such bias because the interviewers were fully trained in distinguishing GT from IBT. However, selection bias may have still occurred, as all subjects were frail and were receiving some level of long-term care. To control for various confounders, multivariable logistic regression was performed.

Although one strength of this study is that we examined the association of GT with a wide range of sociodemographic, health, and socioeconomic factors in the elderly, we did not observe a relationship between GT and any factor other than educational status in this study. However, in a survey targeting 34,374 Japanese elderly people, Ichida et al. [21] showed that people with less than 9 years of schooling were more likely to report poor self-rated health than those with 13 years or more years of schooling. Wang also found that GT was positively associated with general health and mental health among 10,643 Chinese people aged $15-85$ years [26]. On the other hand, Subramanian et al. [27] reported no significant direct association between GT and self-rated health among 21,456 US general residents aged 18 through 89 years, although a complex interaction effect was detected. He indicated that the health-promoting effect of GT was significantly greater for high-trust individuals, whereas the effect of GT on self-rated health was the opposite for lower-trust individuals. They argued that a higher level of GT was associated with a lower probability of reporting poor health. However, our study showed no relationship of GT with self-rated health because our sample size must be smaller than that used in Ichida's, Wang's and Subramanian's surveys. We had another study limitation; the binary-choice format to detect GT was used because the interview to the elderly had to avoid including a large amount of questions, so used the simple format. However, Rotenberg [14], Costa \& McCrae [28], and Yamagishi [22] developed the Likert multi-scale questionnaire to measure GT, respectively, by which we can detect GT in more accurate way. Further studies with a larger sample size using such validated scale measurements are needed.

\section{CONCLUSION}

Our findings suggest that educational status in elderly living at home in a rural region was strongly related to GT, and this finding was explained by the theory of Yamagishi et al. [6,7,22]. A higher educational level might provide subjects with the opportunity to strengthen GT through increased interaction with others. Among the elderly who grew up around the era of Second World War in a rural region in Japan, where high and low levels of education were clearly distinguished, a higher GT was associated with a higher educational status.

\section{ACKNOWLEDGEMENTS}

We express a special gratitude to all the participants of Shin-Hidaka and Hidaka Town, Hokkaido. In particular, we would like to thank Dr. Kishi R., Ms. Katsumata M., Mr. Kamigaki M., Ms. Kakuta S., Mr. Endo T., Mr. Sato N., Ms. Sonoda Y., Ms. Akasaka N., Ms. Kaneko C., Ms. Tsukuda S., Dr. Ishii T., Ms. Ishikawa J., Mr. Saito K., Mr. Goto K., Mr. Tsuboi N., Ms. Aikawa S., Ms. Sado T., Ms. Takei K., Ms. Takeda Y., Ms. Kasai Y., and Ms. Tsuchida K.. The paper was a part of the survey to assess cognitive function among the elderly dwelling at home and was financially supported by the Grant-in-Aid for Scientific Research (C) from Japanese Ministry of Education, Culture, Sports, Science and Technology (No. 20590627).

\section{REFERENCES}

[1] Lewis, J.D. and Weigert, A. (1985) Trust as a social reality. Social Forces, 63, 967-985.

[2] Williamson, O.E. (1993) Calculativeness. Trust and economic organization. Journal of Law and Economics, 30, 131-145.

[3] Zhang, J.X., Zhang, M.Q. and Liang, J. (2000) The role of general trust and specific trust in the path of interper- 
sonal trust model. Acta Psychologica Sinica, 32, 311-316.

[4] Yanagisawa, K., Masui, K., Furutani, K., Nomura, M., Ura, M. and Yoshida, H. (2010) Does higher general trust serve as a psychosocial buffer against social pain? An NIRS study of social exclusion. Social Neuroscience, 6 , 190-197.

[5] Zak, P.J. and Fakhar, A. (2006) Neuroactive hormones and interpersonal trust: International evidence. Economics and Human Biology, 4, 412-429. doi:10.1016/j.ehb.2006.06.004

[6] Yamagishi, T. and Yamagishi, M. (1994) Trust and commitment in the United States and Japan. Motivation and Emotion, 18, 129-166.

[7] Yamagishi, T. and Kikuchi, M. (1999) Trust, gullibility, and social intelligence. Asian Journal of Social Psychology, 2, 145-161. doi:10.1111/1467-839X.00030

[8] Mayer, R.C., Davis, J.H. and Schoorman, F.D. (1995) An integrative model of organizational trust. Academy of Management Review, 20, 709-734.

[9] McAllister, D.J. (1995) Affect- and cognition-based trust as foundations for interpersonal co-operation in organizations. The Academy of Management Journal, 38, 24-59. doi:10.2307/256727

[10] Morrow, J.L. Jr., Hansen, M.H. and Pearson, A.W. (1994) The cognitive and affective antecedents of general trust within cooperative organizations. Journal of Managerial Issues, 16, 48-64.

[11] Kawachi, I., Kennedy, B.P. and Glass, R. (1999) Social capital and self-rated health: A contexual analysis. American Journal of Public Health, 89, 1187-1193. doi:10.2105/AJPH.89.8.1187

[12] Lindström, M. (2008) Social capital, anticipated ethnic discrimination and self-reported psychological health: A population-based study. Social Science \& Medicine, 66, 1-13. doi:10.1016/j.socscimed.2007.07.023

[13] Yipa, W., Subramaniana, S.V., Mitchella, A.D., Leeb, D.T.S., Wangc, J. and Kawachi, I. (2007) Does social capital enhance health and well-being? Evidence from rural China. Social Science \& Medicine, 64, 35-49. doi:10.1016/j.socscimed.2006.08.027

[14] Rotenberg, K.J. (1990) A measure of the trust beliefs of elderly individuals. International Journal of Aging and Human Development, 30, 141-152. doi:10.2190/0P4L-EYW9-2JQ1-WBQK

[15] Geoge, L.K., Landarman, R. and Blazer, D.G. (1991) Cognitive impairment. Free Press, New York.

[16] Tombaugh, T.N. and McIntyre N.J. (1992) The mini- mental state examination: A comprehensive review. Journal of the American Geriatrics Society, 40, 922-935.

[17] Hennessy, C.H., Moriarty, D.G., Zack, M.M., Scherr, P.A. and Brackbill, R. (1994) Measuring health-related quality of life for public health surveillance. Public Health Reports, 109, 665-672.

[18] Folstein, M.F., Folstein, S.E. and Mchugh, P.R. (1975) "Mini-mental state". A practical method for grading the cognitive state of patients for the clinician. Journal of Psychiatric Research, 12, 189-198. doi:10.1016/0022-3956(75)90026-6

[19] Zung, W.W., Richards, C.B. and Short, M.J. (1965) Selfrating depression scale in an outpatient clinic. Further validation of the SDS. Archives of General Psychiatry, 13, 508-515. doi:10.1001/archpsyc.1965.01730060026004

[20] Sherer, M. and Adams, C.H. (1983) Construct validation of the self-efficacy scale. Psychological Reports, 53, 899. 902. doi:10.2466/pr0.1983.53.3.899

[21] Ichida, Y., Kondo, K., Hirai, H., Hanibuchi, T., Yoshikawa, G. and Murata, C. (2009) Social capital, income inequality and self-rated health in Chita peninsula, Japan: A multilevel analysis of older people in 25 communities. Social Science \& Medicine, 69, 489-499. doi:10.1016/j.socscimed.2009.05.006

[22] Yamagishi, T. (1998) The structure of trust. University of Tokyo Press, Tokyo.

[23] Hofstede, G. (1980) Culture's consequences. Sega, Beverly Hills.

[24] Akerlof, G. (1970) The market for "lemons": Qualitative uncertainty and the market mechanism. Quarterly Journal of Economics, 84, 488-500. doi:10.2307/1879431

[25] Fukuyama, F. (1995) Trust: The social virtues and the creation of prosperity. Free Press, Glencoe.

[26] Wang, H., Schlesinger, M. and Hsiao, W.C. (2009) The flip-side of social capital: The distinctive influences of trust and mistrust on health in rural China. Social Science \& Medicine, 68, 133-142. doi:10.1016/j.socscimed.2008.09.038

[27] Subramanian, S.V., Kim, D.J. and Kawachi, I. (2002) Social trust and self-rated health in US communities: A multilevel analysis. Journal of Urban Health, 79, S21-34. doi:10.1093/jurban/79.suppl 1.S21

[28] Costa, P.T. and McCrae, R.R. (1992) Revised NEO personality inventory and NEO five-factor inventory: Professional manual. Psychological Assessment Resources. 